\title{
Sol-Gel Transitions of Linear Low Density Polyethylenes in Organic Solvents
}

\author{
Masaru OKABE, ${ }^{*}$ Munetoshi IsAyama, ${ }^{* *}$ and Hideomi MatsudA** \\ *Department of Chemical Process Engineering, Faculty of Engineering, \\ Ikutoku Technical University, \\ 1030, Shimoogino, Atsugi, Kanagawa 243-02, Japan \\ **Department of Chemical Engineering, Faculty of Textile Science, \\ Shinshu University, 3-15-1, Tokida, Ueda, Nagano 386, Japan
}

(Received April 19, 1984)

\begin{abstract}
The sol-gel transitions of linear low density polyethylenes in decalin, tetralin, $o$ xylene, and toluene were studied. Each sample was a copolymer of ethylene and butene-1, 4-methylpentene-1, or octene-1. A thermo-reversible gel was formed for each system and gel-melting temperatures were measured at concentrations of about $4-14 \mathrm{~g} / 100 \mathrm{~cm}^{3}$. It was found experimentally that the gel-melting temperature depended on polymer concentration, molecular weight of the polymer, and the kind of comonomer. Moreover, the structure of crystallite involved in the gel was examined by a polarizing microscope and many spherulites were observed. The data were treated phenomenologically using the thermodynamic theory of Takahashi, Nakamura, and Kagawa, derived for the gel-melting temperature of a crystalline linear copolymer gel. In each solvent, a linear relationship was found to hold between the reciprocal absolute gel-melting temperature $1 / T_{\mathrm{m}}^{\mathrm{g}}$ and $\ln V_{2} N$ for samples having a same kind of comonomer, where $V_{2}$ is the volume fraction of the polymer and $N$ is the weight-average degree of polymerization.

KEY WORDS Sol-Gel Transition / Gel / Polyethylene / Linear Low Density

Polyethylene / Gel-Melting Temperature / Spherulite /
\end{abstract}

It is well known that some crystalline copolymers or crystalline polymers with copolymeric character form thermo-reversible gels from their solutions on cooling and the gels convert to solutions on warming. ${ }^{1}$ In previous papers, ${ }^{2-5}$ we discovered that a branched low density polyethylene, which had both short and long chain branches in a molecule and thus considered as a crystalline copolymer, formed a thermo-reversible gel in decalin, tetralin, $o$-xylene, toluene, benzene, and cyclohexane-carbon disulfide mixed solvent without precipitating into crystalline phase. In those studies, we measured the gel-melting temperature of a branched low density polyethylene gel at a wide range of polymer concentration and it was found experimentally that the gel-melting temperature $T_{\mathrm{m}}^{\mathrm{g}}$ increased slowly with increasing polymer concentration and the data satisfied well the empirical relation of Eldridge and Ferry ${ }^{6}$ : the reciprocal absolute gel-melting temperature $1 / T_{\mathrm{m}}^{\mathrm{g}}$ varied linearly with the logarithm of volume fraction of the polymer $\ln V_{2}$. Moreover, we applied the theory of Takahashi, Sakai, and Kato ${ }^{7}$ to our experimental results of gel-melting temperature and made a thermodynamic analysis. ${ }^{4,5,8}$

Recently, linear low density polyethylene, a copolymer of ethylene and alkene such as butene-1, 4-methyl-pentene-1, or octene-1, has been synthesized for practical uses. Since the linear low density polyethylene is also a crystalline copolymer, the polymer in suitable organic solvents should form a thermo-reversible gel on cooling.

A few years ago, Barham, Hill, and Keller 
reported that a dilute solution of polyethylene in xylene converted to gel on cooling, after being stirred at an elevated temperature. ${ }^{9}$ On the other hand, Edwards and Mandelkern reported that a linear low density polyethylene in $p$-xylene, a copolymer of ethylene and butene-1, converted to gel at a dilute concentration range without stirring or agitating the solution. ${ }^{10}$ Independently, we reported experimental studies on the sol-gel transition of linear low density polyethylenes, linear high density polyethylenes, and isotactic polypropylenes in organic solvents. ${ }^{11-13}$

It is considered from these experimental facts that the sol-gel transition of polyolefin in organic solvents is one of the general properties of these polymers. However, the structure in a polyolefin gel and the nature or mechanism of the thermo-reversible sol-gel transition are very complex, and these problems have not been definitely and satisfactorily resolved.

In this paper, we measured gel-melting temperature of linear low density polyethylene gels in decalin, tetralin, $o$-xylene, and toluene, and investigated the dependence of the gelmelting temperature on the polymer concentration, molecular weight of the polymer, and the kind of comonomer of the linear polyethylene. Furthermore, the structure of crystallite involved in the gel was observed using a polarizing microscope.

\section{EXPERIMENTAL}

\section{Materials}

Eleven samples of unfractionated linear low density polyethylene (LLDPE) were used. The samples were supplied and characterized by Motegi of the Ohita Laboratory, Showa Denko Co. The typical physical properties of the samples are shown in Table I. The comonomers of LLDPE-1-4, LLDPE-5-7, and LLDPE-8 - 11 are butene-1, 4-methylpentene-1, and octene-1, respectively. The molecular weights of LLDPE-1, LLDPE-7, and LLDPE-9 were largest in the samples with the same kind of comonomer. Each sample had a different number of short chain branches per 1000-methylene groups and molecular weights.

Purification of the samples was carried out as follows: Pellets of the LLDPE sample were dissolved completely in purified xylene at its boiling point under a reflux condenser and

Table I. Typical physical properties of linear low density polyethylenes

\begin{tabular}{|c|c|c|c|c|}
\hline Sample & $\frac{\text { Density }}{\mathrm{gcm}^{-3}}$ & $M_{w} \times 10^{-4 \mathrm{a}}$ & Comonomer & $\mathrm{SCB} / 1000 \mathrm{CH}_{2}{ }^{\mathrm{b}}$ \\
\hline LLDPE-1 & 0.915 & 11.3 & Butene-1 & 25.3 \\
\hline LLDPE-2 & 0.920 & 10.4 & Butene-1 & 24.3 \\
\hline LLDPE-3 & 0.930 & 7.1 & Butene-1 & 21.0 \\
\hline LLDPE-4 & 0.918 & 5.5 & Butene-1 & 22.5 \\
\hline LLDPE-5 & 0.916 & 8.0 & 4-Methyl-pentene-1 & 15.3 \\
\hline LLDPE-6 & 0.923 & 8.4 & 4-Methyl-pentene-1 & 10.1 \\
\hline LLDPE-7 & 0.927 & 8.7 & 4-Methyl-pentene-1 & 7.6 \\
\hline LLDPE-8 & 0.923 & 8.3 & Octene-1 & 10.6 \\
\hline LLDPE-9 & 0.917 & 11.9 & Octene-1 & 15.4 \\
\hline LLDPE-10 & 0.913 & 8.0 & Octene-1 & 23.9 \\
\hline LLDPE-11 & 0.923 & 9.0 & Octene-1 & 11.9 \\
\hline
\end{tabular}

a $M_{w}$, the weight-average molecular weight determined by GPC.

b $\mathrm{SCB} / 1000 \mathrm{CH}_{2}$, the number of short chain branches per 1000 -methylene groups determined by ${ }^{13} \mathrm{C}$ NMR. 
precipitated into cooled methanol. After washing with purified methanol, the sample was dried under reduced pressure at $70^{\circ} \mathrm{C}$ until a constant weight was reached. Decalin, tetralin, $o$-xylene, and toluene used as solvents were purified by the ordinary method before use.

\section{Measurements of Gel-Melting Temperature}

Measurements of gel-melting temperature were carried out by the so-called falling-ball method using a small steel ball and a glass tube. A schematic drawing of the apparatus for gel-melting temperature and the size of the glass tube are shown in Figure 1. In our preliminary experiments, the dependence of gel-melting temperature on the weight of the steel ball was examined and about $30 \mathrm{mg}$ weight with $2 \mathrm{~mm}$ in diameter of a steel ball were used in this study.

A LLDPE gel was formed in a glass tube as follows: A definite weight of LLDPE, a definite volume of solvent, and the steel ball were put into the apparatus and the glass tube was sealed. The steel ball was then fixed by a magnet at the upper part of the glass tube. In decalin and tetralin, the polymer was dissolved completely at $180^{\circ} \mathrm{C}$ and in $o$-xylene and toluene at $110^{\circ} \mathrm{C}$ in a glycerin bath. After the solution was maintained at room temperature for $5 \mathrm{~min}$, the apparatus was immersed in a thermobath regulated at $100^{\circ} \mathrm{C}$ for $25 \mathrm{~min}$. Finally, it was placed in a thermobath kept at $10^{\circ} \mathrm{C}$ for $16 \mathrm{~h}$ to make a gel.

During this process, the polymer did not precipitate from the solution and the solution turned into a gel. The steel ball was then carefully moved to the top of the gel by magnetic manipulation as shown in Figure 1, and the gel was warmed very slowly at a rate of $6^{\circ} \mathrm{Ch}^{-1}$. The height of the steel ball from a reference level was measured by a cathetometer at various temperatures. When the gel melted, the steel ball began to fall. Typical examples of measurement of gel-melting temperature are shown in Figure 2. The temperature at which the horizontal and vertical

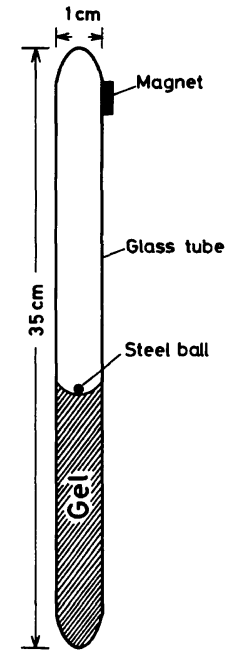

Figure 1. Apparatus for gel-melting temperature.

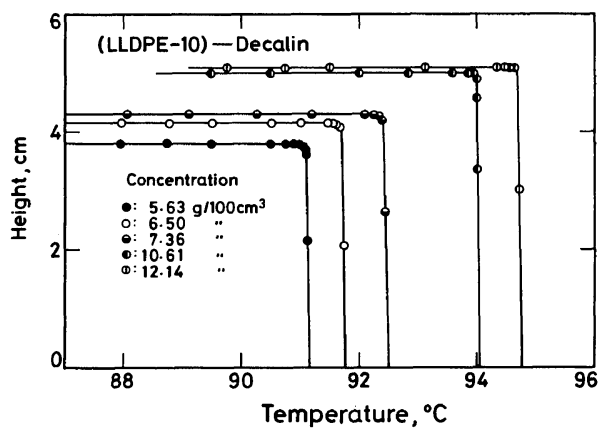

Figure 2. Examples for measurement of gel-melting temperature.

straight lines intersected was taken as the gelmelting temperature at each polymer concentration. The same measurements were repeated five or six times for each concentration, and the reproducibility of the data was within about $\pm 1^{\circ} \mathrm{C}$. An average value was used for subsequent treatment of gel-melting temperature.

Observation of Gel by Polarizing Microscope

Observation of crystallite in a LLDPE gel was carried out using an Olympus Model BHA-P polarizing microscope. The gel was carefully placed on a slide glass of the microscope and a cover glass was put on the gel. The 
crystallite in the gel was then immediately observed and photographed using Fuji Neopan 400 Ultra High Speed Film with crossed nicols.

\section{RESULTS AND DISCUSSION}

Solutions of LLDPE in decalin, tetralin, oxylene, and toluene converted to gels on cooling and the gels converted to solutions on warming. These sol-gel transitions were thermo-reversible. In dilute solutions, no thermoreversible gel was formed by the method shown in the experimental section, but the crystalline phase separation took place on cooling.

When the solutions converted to gels, the gels exhibited considerable turbidity. Therefore, many small crystallites may exist in the gel. The existence of the lamellar crystal with folded chains in crystalline polymers such as polyethylene, polypropylene, and other polymers is well recognized by X-ray investigations of single crystals of these polymers, which can be prepared by cooling dilute solutions and melts. It is considered that, in the fibrils of cellulosic fibers, there are structures such as fringed micelles. The structure of a crystallite in a polymer gel has been conventionally considered to be the fringed micellar type and some thermodynamic theories have been derived for the gel-melting temperature, assuming such a model. ${ }^{1,7}$ We also analyzed our experimental data by the theory ${ }^{7}$ in the previous studies. ${ }^{4,8}$

However, Edwards and Mandelkern reported, on the basis of small-angle X-ray scattering (SAXS) measurement for a linear low density polyethylene gel in $p$-xylene, that the crystallite involved in the gel was not the fringed micellar type, but a lamellar crystallite. ${ }^{10} \mathrm{We}$ also examined the crystallite in the present LLDPE gel using a polarizing microscope. An example of a photograph of the crystallite in the gel is shown in Figure 3 for LLDPE-9 in tetralin at concentration of $5 \mathrm{~g}$ /

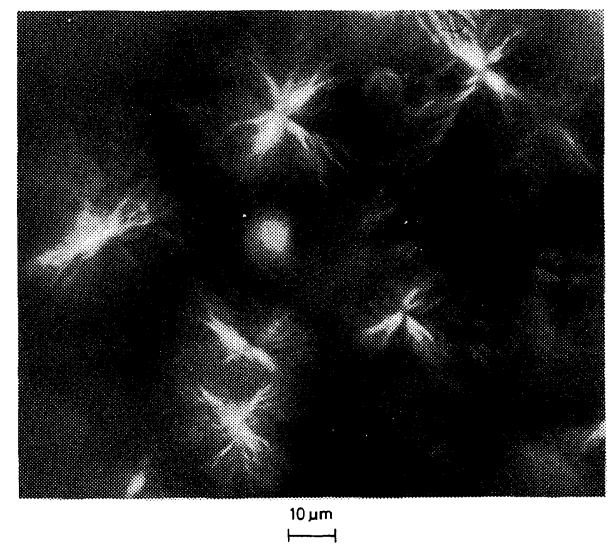

Figure 3. Example of photograph of spherulites in linear low density polyethylene gel by polarizing microscope with crossed nicols: Sample, LLDPE-9; comonomer, octene-1; molecular weight, $M_{w}=11.9 \times$ $10^{4}$; solvent, tetralin; concentration, $5 \mathrm{~g} / 100 \mathrm{~cm}^{3}$.

$100 \mathrm{~cm}^{3}$. Many spherulites were observed in this photograph. Similar photographs were obtained for other samples and solvents. It was definitely found that spherulites exist in a LLDPE gel.

Examples of the relation between gel-melting temperature $T_{\mathrm{m}}^{\mathrm{g}}\left({ }^{\circ} \mathrm{C}\right)$ and polymer concentration in decalin $C\left(\mathrm{~g} / 100 \mathrm{~cm}^{3}\right)$ are shown in Figure 4. Similar relations were also obtained in tetralin, $o$-xylene, and toluene. In Figure 4, the gel-melting temperature increases slowly with increasing polymer concentration, and this tendency is similar to the branched low density polyethylene gel reported in the previous paper. ${ }^{8}$

In samples having identical comonomers, the gel-melting temperature of the sample with the largest molecular weight was the highest. For example, in LLDPE-8-11 whose comonomers are octene-1, the gel-melting temperature of LLDPE-9 was the highest. This gel had the largest molecular weight among four samples. Moreover, the gel-melting temperatures of eleven samples approximately separated into three groups according to three kinds of comonomers in the samples. The highest group of gel-melting temperatures was 
LLDPE-8 - 11 whose comonomers were all octene-1. Thus, the gel-melting temperature of LLDPE depends on the polymer concentration, the molecular weight of the polymer, and the kind of comonomer.

Eldridge and Ferry derived the following relation between absolute gel-melting temperature $T_{\mathrm{m}}^{\mathrm{g}}(\mathrm{K})$ and volume fraction of a polymer $V_{2}$ in a gel, assuming the formation of cross-links to result from binary association of the polymer chains ${ }^{6}$ :

$$
\ln V_{2}=\text { const. }+\Delta H_{\mathrm{m}} / R T_{\mathrm{m}}^{\mathrm{g}}
$$

where $\Delta H_{\mathrm{m}}$ is the heat absorbed on formation of one mol of junction points and $R$ is the gas constant. They then calculated the values of $\Delta H_{\mathrm{m}}$ for gelatin gels by eq 1 .

According to eq 1, a plot of $\ln V_{2}$ vs. $1 / T_{\mathrm{m}}^{\mathrm{g}}$ should yield a straight line. Examples of Eldridge and Ferry's plot for LLDPE gels in decalin are illustrated in Figure 5. Good straight lines were obtained for all samples and similar tendencies were also obtained in tetralin, $o$-xylene, and toluene. Considering the linear relationship obtained between $\ln V_{2} v s$. $1 / T_{\mathrm{m}}^{\mathrm{g}}$ as shown in Figure 5, the dependence of the gel-melting temperature of a LLDPE gel on polymer concentration can be represented well by this type of plot. However, any useful information regarding the dependence of gelmelting temperature on the kind of comonomer or molecular weight could not be obtained by eq 1 , since, as pointed out by Takahashi et $a l .{ }^{7}$ eq 1 is merely empirical.

Since a LLDPE is a crystalline copolymer, no such single cross-links formed by binary associations between polymer chains as assumed by Eldridge and Ferry, but rather multiple cross-links formed by many small crystallites should exist in a LLDPE gel in addition to lamellae or spherulites. The fringed micellar crystallites form a net-work in the LLDPE gel and behave as cross-linking junctions.

Recently, Takahashi, Nakamura, and Kagawa ${ }^{1}$ treated gel formation as crystallization and derived the thermodynamic theory

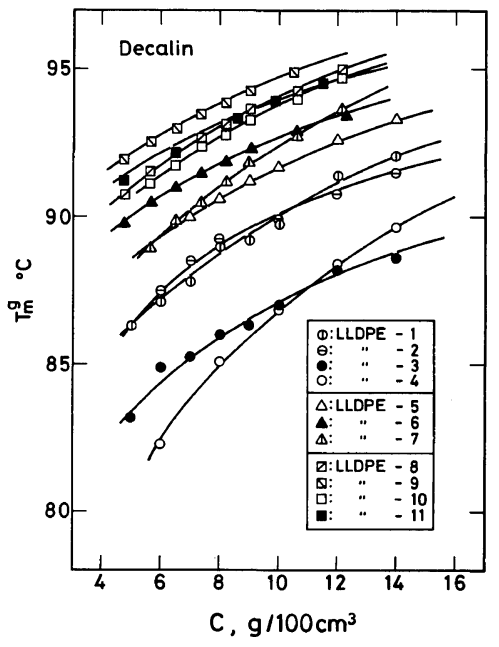

Figure 4. Relation between gel-melting temperature $T_{\mathrm{m}}^{\mathrm{g}}\left({ }^{\circ} \mathrm{C}\right)$ and polymer concentration $\mathrm{C}\left(\mathrm{g} / 100 \mathrm{~cm}^{3}\right)$ in the gel for decalin system.

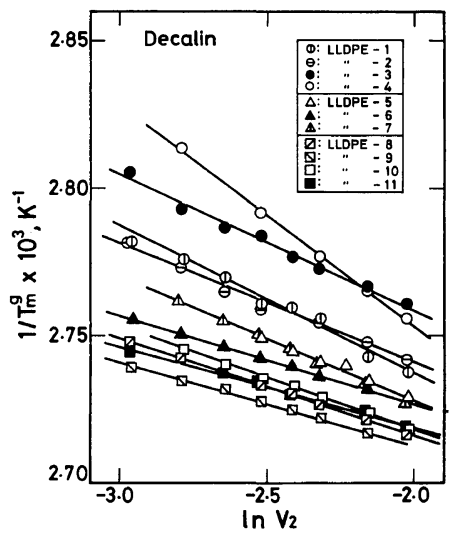

Figure 5. Relation between the reciprocal of gelmelting temperature $1 / T_{\mathrm{m}}^{\mathrm{g}}\left(\mathrm{K}^{-1}\right)$ and the logarithm of volume fraction of polymer $\ln V_{2}$ for decalin system by eq 1 .

for gel-melting temperature of a crystalline linear copolymer gel from the melting point depression of a copolymer-diluent system as formulated by Flory ${ }^{14}$ and Mandelkern, ${ }^{15}$ assuming the cross-links to be crystallites comprised of $\zeta$ units in length and $\rho$ crystalline sequences in cross section, as in the case of the fringed micellar structure. They estimated the size of the crystallite $\zeta$ in thermally reversible gels of poly(vinyl chloride), ${ }^{1}$ ethylene-vinyl 
acetate copolymer, ${ }^{16}$ poly(vinyl alcohol), ${ }^{17}$ and cellulose diacetate. ${ }^{18}$

The LLDPE gel actually includes spherulites composed of the lamella, and thus their theory may not be rigorously applicable to this gel. There is no theory to interpret satisfactorily the gel-melting temperature of a polyolefin gel in organic solvents, where spherulites exist. Consequently, we utilize the theory of Takahashi, Nakamura, and Kagawa to treat our experimental results on gel-melting temperatures, as follows:

$$
\begin{aligned}
\frac{1}{T_{\mathrm{m}}^{\mathrm{g}}}= & \frac{\zeta}{\zeta \Delta h_{\mathrm{u}}+\zeta B^{\prime} V_{\mathrm{A}}-2 \sigma_{\mathrm{ec}}} \\
& \times\left(\frac{\Delta h_{\mathrm{u}}}{T_{\mathrm{m}}^{0}}+\frac{R V_{\mathrm{A}}}{V_{1}}-R \ln X_{\mathrm{A}}\right) \\
& -\frac{R}{\zeta \Delta h_{\mathrm{u}}+\zeta B^{\prime} V_{\mathrm{A}}-2 \sigma_{\mathrm{ec}}} \ln V_{2} N
\end{aligned}
$$

where $T_{\mathrm{m}}^{\mathrm{g}}$ is the observed gel-melting temperature of a crystalline linear copolymer gel, $\zeta$, the ethylene-unit length in a crystallite, $\Delta h_{\mathrm{u}}$, the heat of fusion of an ethylene unit, $B^{\prime}$, the cohesive energy density defined by $\chi_{1}=B^{\prime} V_{1} /$ $R T, \chi_{1}$, the interaction parameter between polymer and solvent, $V_{1}$ and $V_{\mathrm{A}}$, the molar volumes of solvent and a crystalline unit, respectively, $\sigma_{\text {ec }}$, the end interfacial free energy per crystalline sequence, $T_{\mathrm{m}}^{0}$, the melting temperature of the polymer, $R$, the gas constant, $X_{\mathrm{A}}$, the mole fraction of crystalline units, $V_{2}$, the volume fraction of the polymer in the gel, and $N$, the weight-average degree of polymerization of the polymer.

In our phenomenological treatment, it is no purpose to estimate the values of the molecular and thermodynamic parameters involved in eq 2. Thus, eq 2 can be written simply as

$$
1 / T_{\mathrm{m}}^{\mathrm{g}}=A-B \cdot \ln V_{2} N
$$

In this study, we use eq 3 to treat our experimental data. Examples of the plot of $1 / T_{\mathrm{m}}^{\mathrm{g}} v s$. $\ln V_{2} N$ by eq 3 are shown in Figures 6-9. Three straight lines with different gradients were obtained in each solvent depending on

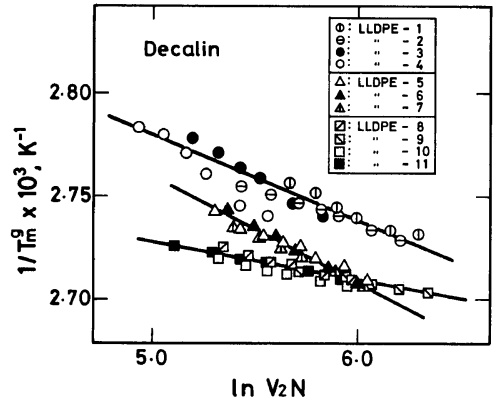

Figure 6. Relation between the reciprocal of gelmelting temperature $1 / T_{\mathrm{m}}^{\mathrm{g}}\left(\mathrm{K}^{-1}\right)$ and $\ln V_{2} N$ for decalin system by eq 3 .

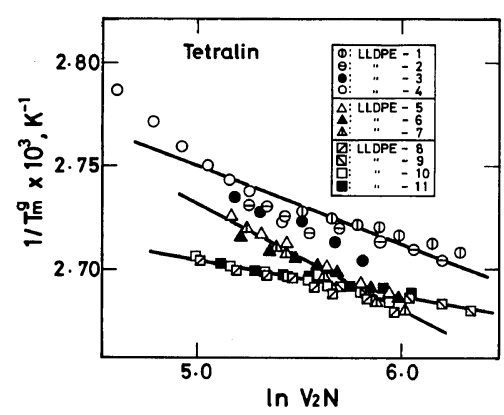

Figure 7. Relation between the reciprocal of gelmelting temperature $1 / T_{\mathrm{m}}^{\mathrm{g}}\left(\mathrm{K}^{-1}\right)$ and $\ln V_{2} N$ for tetralin system by eq 3 .

three kinds of comonomers: butene-1, 4methyl-pentene-1, and octene-1. Judging from our treatment of the present experimental data by eq 3 , the gel-melting temperature $T_{\mathrm{m}}^{\mathrm{g}}$ considerably depends on the kind of comonomer of the linear polyethylene and the plot of $1 / T_{\mathrm{m}}^{\mathrm{g}}$ vs. $\ln V_{2} N$ gave a common straight line when the samples all had the same kind of comonomer.

In our preliminary experiments, we measured the gel-melting temperatures of LLDPE gels formed by two methods: the cooling of a LLDPE solution rapidly or very slowly. The gel-melting temperature of the former gel was higher than that of the latter gel at same concentration and this tendency became more pronounced at the lower concentration region. The spherulites involved in the former gel were 
considerably smaller than those in the latter gel. Moreover, at a low concentration of about $1 \mathrm{~g} / 100 \mathrm{~cm}^{3}$, the solution did not convert to gel by the latter method and phase separation took place, but did so by the former method. Then, many large spherulites were observed when phase separation took place. Thus, growth of the spherulites decreases the cross-linking loci of net-works in a polyethylene gel. Of course, as the present LLDPE gel was formed by rapid cooling, a high content of amorphous chains also exist in the gel besides crystallites. Thus, tie chains may exist between lamellae or spherulites. These tie chains and crystallites show fringed micellar-like behavior. Though the structure in the LLDPE gel is not clear, the plot of Takahashi, Nakamura, and Kagawa may indicate that fringed micellar crystallites behave as cross-linking junctions of net-works in the gel. However, a morphological study is necessary to clarify the gel structure in detail. There is no theory to interpret completely the gel-melting temperature of a polyolefin gel. Consequently, only phenomenological treatments were carried out in this paper.

The following results were obtained for solgel transitions of linear low density polyethylenes in organic solvents:

1. Solutions of linear low density polyethylenes in decalin, tetralin, $o$-xylene, and toluene convert to gels on cooling, which in turn convert to solutions on warming. These sol-gel transitions are thermo-reversible.

2. Spherulites are observed in the gel through a polarizing microscope, indicating the crystallites in the gel to be composed of lamella.

3. The gel-melting temperature depends on polymer concentration, molecular weight, and the kind of comonomer. The reciprocal absolute gel-melting temperature $1 / T_{\mathrm{m}}^{\mathrm{g}}$ has a linear relationship to the logarithm of volume fraction of the polymer in the gel $\ln V_{2}$ and the dependence of gel-melting temperature on the polymer concentration can be represented well by Eldridge and Ferry's type of plots.

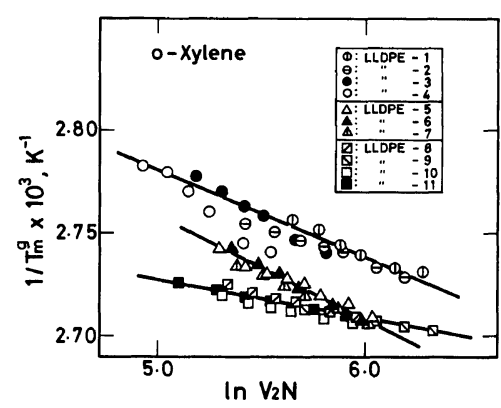

Figure 8. Relation between the reciprocal of gelmelting temperature $1 / T_{\mathrm{m}}^{\mathrm{g}}\left(\mathrm{K}^{-1}\right)$ and $\ln V_{2} N$ for $o$-xylene system by eq 3 .

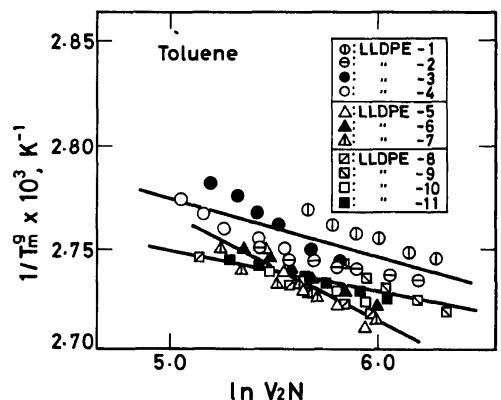

Figure 9. Relation between the reciprocal of gelmelting temperature $1 / T_{\mathrm{m}}^{\mathrm{g}}\left(\mathrm{K}^{-1}\right)$ and $\ln V_{2} N$ for toluene system by eq 3 .

4. The dependence of gel-melting temperature on the kind of comonomer can be interpreted well by the theory of Takahashi, Nakamura, and Kagawa. The plot of $1 / T_{\mathrm{m}}^{\mathrm{g}} v s$. $\ln V_{2} N$ by their theory gave a common straight line, independent of the molecular weight of the polymer, when the samples all had the same kind of comonomer. Otherwise, another straight line was obtained depending considerably on the comonomer. The plot of Takahashi, Nakamura, and Kagawa may indicate that fringed micellar or micellar-like crystallites behave as cross-linking junctions of networks in a linear low density polyethylene gel, though the spherulites exist in the gel.

Acknowledgement. The authors wish to express their sincere appreciation to $\mathrm{Mr}$. Yoshihiro Motegi of the Ohita Laboratory, 
Showa Denko Co. for supplying and characterizing the linear low density polyethylene samples. One of the authors ( $H$. Matsuda) should like to thank Showa Denko Co. for a research grant which made this work possible. This work was also partly supported by a Grant-in-Aid for Scientific Research from the Ministry of Education, Science, and Culture of Japan.

\section{REFERENCES}

1. A. Takahashi, T. Nakamura, and I. Kagawa, Polym. J., 3, 207 (1972).

2. H. Matsuda, K. Araki, M. Yamada, and S. Kuroiwa, Rep. Prog. Polym. Phys. Jpn., 20, 69 (1977).

3. H. Matsuda and S. Kuroiwa, Rep. Prog. Polym. Phys. Jpn., 22, 45 (1979).

4. H. Matsuda, K. Araki, H. Fujimatsu, and S. Kuroiwa, Polym. J., 13, 587 (1981).

5. H. Matsuda, H. Fujimatsu, M. Imaizumi, and S. Kuroiwa, Polym. J., 13, 807 (1981).
6. J. E. Eldridge and J. D. Ferry, J. Phys. Chem., 58, 992 (1954).

7. A. Takahashi, M. Sakai, and T. Kato, Polym. J., 12, 335 (1980).

8. H. Matsuda, M. Imaizumi, H. Fujimatsu, S Kuroiwa, and M. Okabe, Polym. J., 16, 151 (1984).

9. P. J. Barham, M. J. Hill, and A. Keller, Colloid Polym. Sci., 258, 899 (1980).

10. C. O. Edwards and L. Mandelkern, J. Polym. Sci., Polym. Lett. Ed., 20, 355 (1982).

11. H. Matsuda, H. Fujimatsu, M. Imaizumi, and S. Kuroiwa, Polym. Prepr. Jpn., 30, 575 (1981).

12. H. Matsuda, M. Imaizumi, H. Fujimatsu, and S. Kuroiwa, Rep. Prog. Polym. Phys. Jpn., 24, 41 (1981).

13. H. Matsuda, M. Imaizumi, M. Isayama, $\mathrm{H}$ Fujimatsu, and S. Kuroiwa, Rep. Prog. Polym. Phys. Jpn., 25, 87 (1982).

14. P. J. Flory, J. Chem. Phys., 17, 223 (1949).

15. L. Mandelkern, J. Appl. Phys., 26, 443 (1955).

16. A. Takahashi, Polym. J., 4, 379 (1973).

17. A. Takahashi and S. Hiramitsu, Polym. J., 6, 103 (1974).

18. A. Takahashi, T. Kawaharada, and T. Kato, Polym. J., 11, 671 (1979). 